\title{
Tratamento com Stent em Tronco de Artéria Coronária Esquerda por Compressão do Tronco da Artéria Pulmonar em Paciente com Hipertensão Pulmonar Esquistossomótica
}

\author{
Adriano Assis Mendes¹, Flávio A. A. Japyassu², Flávio Roberto², Diana Lamprea', Eugênio Albuquerque, \\ Carlos G. P. Roncal', Henrique Q. Cartaxo ${ }^{3}$, Suela D. Farias ${ }^{2}$
}

\section{RESUMO}

A hipertensão arterial pulmonar frequentemente está associada a dor torácica com características anginosas e sua etiologia é desconhecida. A compressão extrínseca do tronco de artéria coronária esquerda pela artéria pulmonar é uma causa tratável e deve ser considerada. É apresentado o caso de uma paciente com hipertensão arterial pulmonar de etiologia esquistossomótica, com angina do peito decorrente de compressão do tronco de artéria coronária esquerda, que foi tratada com stent intracoronário. Os sinais e sintomas isquêmicos foram completamente resolvidos e a ultrassonografia intracoronária, realizada na evolução tardia, mostrou a consolidação do resultado angiográfico.

DESCRITORES: Hipertensão pulmonar. Vasos coronários. Stents. Esquistossomose.

\begin{abstract}
Stent Treatment for a Left Main Coronary Artery Compression by a Dilated Pulmonary Trunk in a Patient with Schistosomotic Pulmonary Hypertension

Pulmonary hypertension is frequently associated to chest pain with anginous characteristics and its etiology is uncertain. Extrinsic compression of the left main coronary artery by the pulmonary artery is a treatable cause and should be taken into consideration. We present the case of a patient with pulmonary hypertension of schistosomotic etiology presenting angina due to compression of the left main coronary artery, who was treated with an intracoronary stent. Signs and symptoms of ischemia were completely resolved and a late follow-up intravascular ultrasound confirmed the vessel patency.
\end{abstract}

KEY-WORDS: Pulmonary hypertension. Coronary vessels. Stents. Schistosomiasis.

\begin{abstract}
A hipertensão arterial pulmonar é uma doença grave da circulação pulmonar, que frequentemente progride para falência ventricular direita e morte. ${ }^{1}$ Um dos principais sintomas é a dor torácica, ocorrendo em torno de $41 \%$ dos casos de hipertensão arterial pulmonar idiopática. ${ }^{2}$ Várias teorias têm sido especuladas para esse quadro, entre elas a dilatação dolorosa da artéria pulmonar ${ }^{3}$ e a isquemia ventricular direita. ${ }^{4}$
\end{abstract}

1 PROCAPE - Pronto Socorro Cardiológico de Pernambuco - Recife, PE, Brasil.

2 Hospital Memorial São José - Recife, PE, Brasil.

3 Hospital Português - Recife, PE, Brasil.

Correspondência: Adriano Assis Mendes. PROCAPE - Pronto Socorro

Cardiológico de Pernambuco. Rua dos Palmares, s/n - Santo Amaro

- Recife, PE, Brasil - CEP 50100-060

E-mail: willow@hotlink.com.br

Recebido em: 26/10/2009 • Aceito em: 17/2/2010
A infestação humana pelo Schistosoma ocorre no Brasil de forma endêmica e o cor pulmonale esquistossomótico tem sido considerado condição clínica importante, cuja incidência varia nas diferentes séries entre $11 \%$ e $33 \%$, em autópsias de pacientes com comprometimento hepatoesplênico. ${ }^{5,6}$ Nos casos de hipertensão arterial pulmonar associada à esquistossomose, que evoluem para cor pulmonale, a incidência varia entre $1,5 \%$ e $9,6 \% .^{7,8}$

Pelo fato de o Pronto Socorro Cardiológico de Pernambuco (PROCAPE) da Universidade de Pernambuco ser um centro nacional de referência no estudo da hipertensão arterial pulmonar, foi possível diagnosticar um número maior de casos em toda a região. Apresentamos o caso de paciente com hipertensão arterial pulmonar de etiologia esquistossomótica, com angina do peito atribuída à oclusão completa do tronco de coronária esquerda por dilatação do tronco de artéria 
pulmonar, diagnosticado por meio de tomografia coronária, assim como seu tratamento com stent e os resultados da ultrassonografia intracoronária, na avaliação angiográfica tardia.

\section{CASO CLÍNICO}

Paciente do sexo feminino, 58 anos de idade, procedente e residente em zona endêmica para esquistossomose, procurou a emergência cardiológica do PROCAPE, em maio de 2007, com queixa de dor torácica em aperto, com irradiação para membro superior esquerdo, em repouso, associada a dispneia, com cerca de três horas de duração. O alívio da dor ocorreu com o uso de nitrato sublingual. A paciente relatou que dois meses antes da internação não apresentava qualquer queixa, quando iniciou dispneia aos médios esforços. Ao interrogatório anamnésico, negou diabetes, mas referiu hipertensão arterial sistêmica controlada com dieta.

À inspeção, apresentava-se dispneica (+++/4+), anictérica, com edema de membros inferiores, bilateralmente $(++/ 4+)$, estase jugular significativa e leve cianose periférica. Ao exame físico, os achados consistiram de: pulmões limpos; ritmo cardíaco regular, em dois tempos, e presença de hiperfonese de P2, com sopro sistólico em foco tricúspide $(+++/ 4+)$. Sua pressão arterial era de 110 × 60 mmHg e a frequência cardíaca era de 56 bpm.

Ao eletrocardiograma, foram identificados: ritmo cardíaco regular sinusal, eixo +120 graus, aumento da amplitude de ondas $\mathrm{R}$ em precordiais direitas e presença de ondas $T$ negativas em paredes anterior, lateral e inferior. A radiografia de tórax revelou leve cardiomegalia, abaulamento do arco médio da pulmonar e redução da vascularidade de ambos os pulmões.

O ecocardiograma revelou ventrículo esquerdo de tamanho normal com função sistólica preservada, mas significativa dilatação de câmaras direitas, associada a dilatação expressiva do tronco $(5,1 \mathrm{~cm})$ e ramos da artéria pulmonar com pressão sistólica da artéria pulmonar, estimada pelo refluxo tricúspide, de $131 \mathrm{mmHg}$. As concentrações das enzimas cardíacas estavam dentro dos limites de normalidade e a ultrassonografia abdominal revelou fibrose periportal.

A paciente foi submetida a estudo hemodinâmico, cujos parâmetros diagnosticados foram: pressão média de átrio direito de $7 \mathrm{mmHg}$, pressão em artéria pulmonar média igual a $53 \mathrm{mmHg}$, índice cardíaco de $3,9 \mathrm{l} / \mathrm{min} / \mathrm{m}^{2}$, resistência pulmonar total de $809 \mathrm{din} / \mathrm{s} / \mathrm{cm}^{5}$ e resistência vascular pulmonar de 641,3 dynes $/ \mathrm{s} / \mathrm{cm}^{5}$. O teste de vasorreatividade pulmonar com óxido nítrico (40 ppm) foi negativo. O estudo angiográfico revelou coronárias livres de ateromatose, tronco de coronária esquerda não visualizado, presença de circulação colateral da artéria coronária direita para a artéria descendente anterior e circunflexa com morfologia "em ponta de lápis" (Figura 1). Submetida a tomografia computadorizada cardíaca multislice, foi possível constatar dilatação significativa do tronco de artéria pulmonar com compressão extrínseca do tronco de coronária esquerda (Figura 2).

Após a internação, a paciente evoluiu com angina em repouso, apesar da medicação, tendo sido indicada revascularização da artéria coronária esquerda, em regime de urgência. Em decorrência do elevado risco cirúrgico, considerando a não resposta ao tratamento clínico, e por tratar-se de tronco de coronária esquerda "protegido" por circulação colateral, optou-se pelo implante de stent convencional (Liberté 4,5 x 16 mm, com pressão de 18 atm) em tronco de coronária esquerda, obtendo-se fluxo TIMI 3 e resolução dos sintomas (Figura 3).

Antecedendo a alta hospitalar, a paciente foi submetida a teste de caminhada de seis minutos, alcançando $345 \mathrm{~m}$ sem queixas e completamente livre de angina. A paciente teve alta hospitalar em uso de sildenafil (150 mg/dia), digital, diurético e ticlopidina (250 mg duas vezes por dia), para uso por seis meses.

$\mathrm{Na}$ avaliação cardiológica, decorridos seis meses do procedimento, a paciente permanecia livre de angina, sendo classificada na classe funcional II. Avaliação angiográfica associada à realização de ultrassom intracoronário constatou stent pérvio, sem sinais de aterosclerose ou trombose (Figura 4).

\section{DISCUSSÃO}

A angina do peito é um sintoma presente em aproximadamente $41 \%$ dos pacientes com hipertensão arterial pulmonar, não estando sua etiologia completamente esclarecida. ${ }^{2}$ Os mecanismos envolvidos seriam: isquemia subendocárdica da parede do ventrículo direito, causada pela compressão intramiocárdica das arteríolas; redução do gradiente de perfusão coronário, decorrente da elevação da pressão no átrio direito; ${ }^{4}$ dilatação aguda do tronco de artéria pulmonar em decorrência de aumentos transitórios da pressão pulmonar; ${ }^{3}$ ou, ainda, compressão extrínseca da artéria coronária esquerda pela dilatação do tronco de artéria pulmonar. ${ }^{9,10}$ Por se tratar de pacientes jovens, sem fatores de risco cardiovascular, a aterosclerose coronária é pouco provável como causa de angina, nesses pacientes.

Em 1957, Corday et al., ${ }^{11}$ ao relatar pioneiramente esse quadro clínico, sugeriram que a compressão do tronco de coronária esquerda, decorrente da dilatação do tronco de artéria pulmonar, podia explicar os sintomas de insuficiência coronária em pacientes com hipertensão arterial pulmonar. Em 1997, foi publicado o primeiro caso de compressão extrínseca do tronco de coronária esquerda por dilatação do tronco de artéria pulmonar em paciente com hipertensão arterial pulmonar primária. ${ }^{10} \mathrm{Em}$ 2006, Piña et al., ${ }^{12}$ ao rever 
Mendes AA, et al. Tratamento com Stent em Tronco de Artéria Coronária Esquerda por Compressão do Tronco da Artéria Pulmonar em Paciente com Hipertensão Pulmonar Esquistossomótica. Rev Bras Cardiol Invasiva. 2010;18(1):89-94.

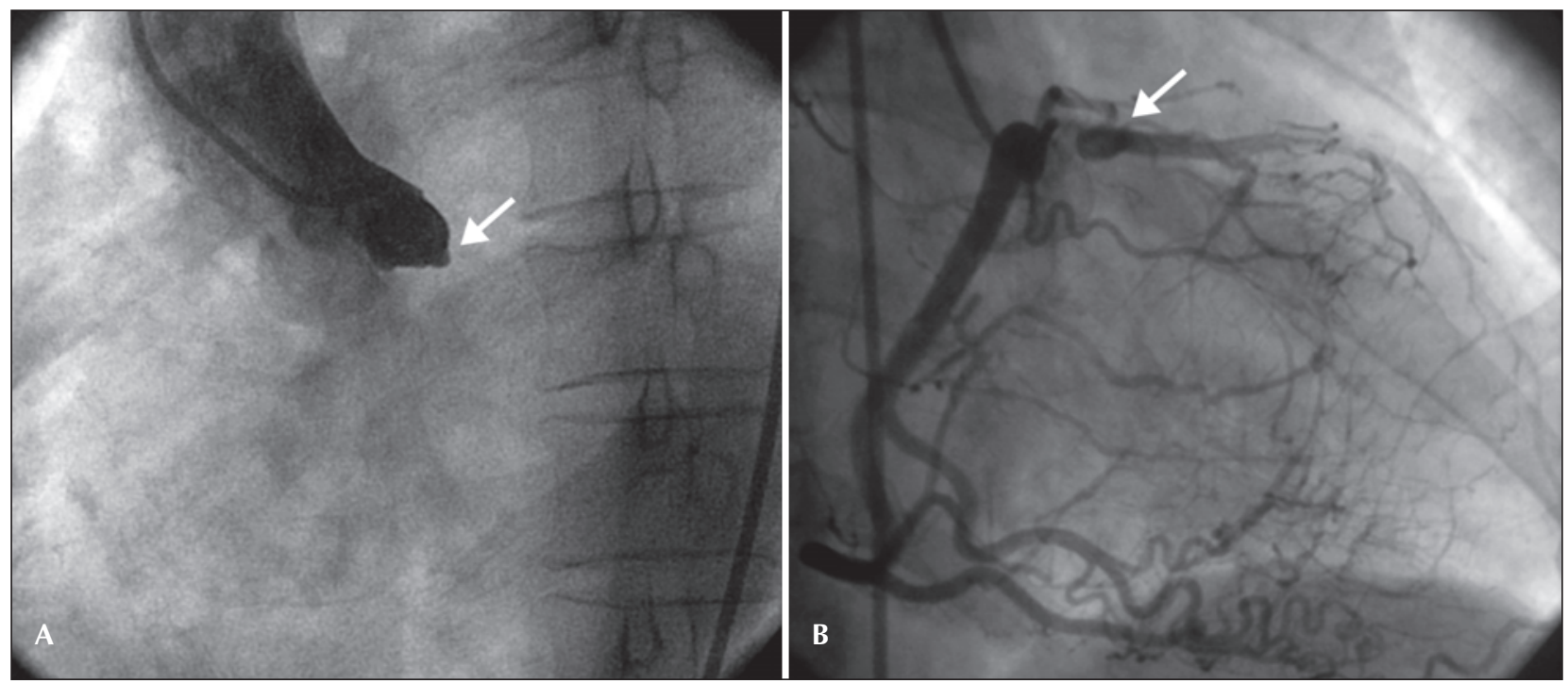

Figura 1 - Em A, injeção de contraste na aorta com ausência da visualização do tronco de coronária esquerda (seta). Em B, circulação colateral da artéria coronária direita para a artéria descendente anterior e artéria circunflexa, com morfologia "em ponta de lápis" (seta).
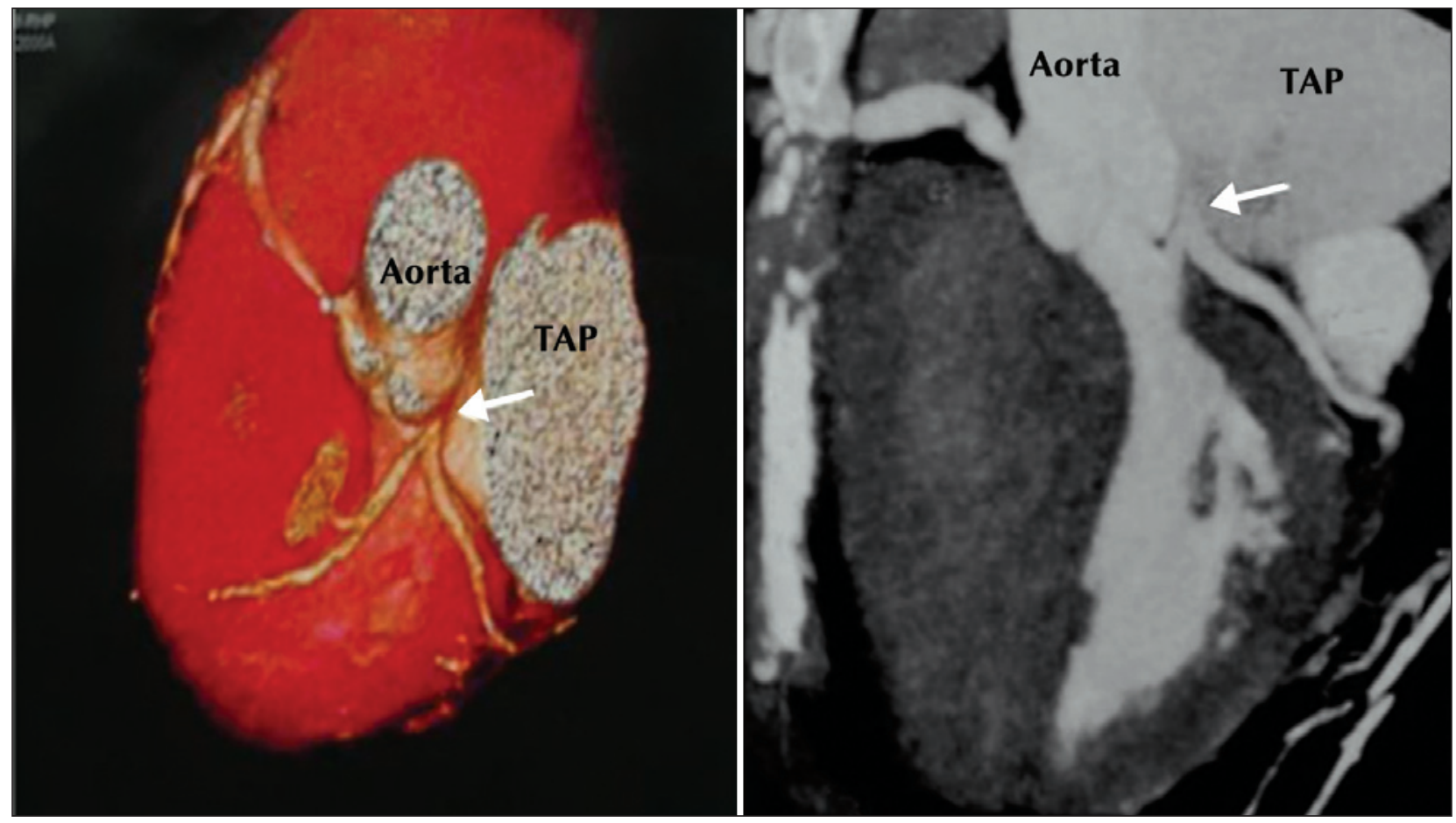

Figura 2 - Em A, ausência de visualização do tronco de coronária esquerda (seta). Em B, dilatação do tronco da artéria pulmonar, com compressão extrínseca do tronco de coronária esquerda (seta). TAP = tronco da artéria pulmonar.

45 casos de compressão coronária, constataram que a maioria estava associada a cardiopatia congênita acianótica ou a hipertensão arterial pulmonar idiopática. Nesse mesmo estudo, ${ }^{12}$ os autores identificaram apenas um caso de compressão da artéria coronária esquerda por hipertensão arterial pulmonar de etiologia esquistossomótica. ${ }^{13}$ Apesar de apresentarem diferen- tes graus de hipertensão pulmonar, o fator determinante para a compressão coronária foi o diâmetro do tronco de artéria pulmonar. ${ }^{14}$

O caso apresentado representa o primeiro, na literatura, de compressão total do tronco de coronária esquerda pelo tronco de artéria pulmonar em pacientes com hipertensão arterial pulmonar esquistossomótica. 

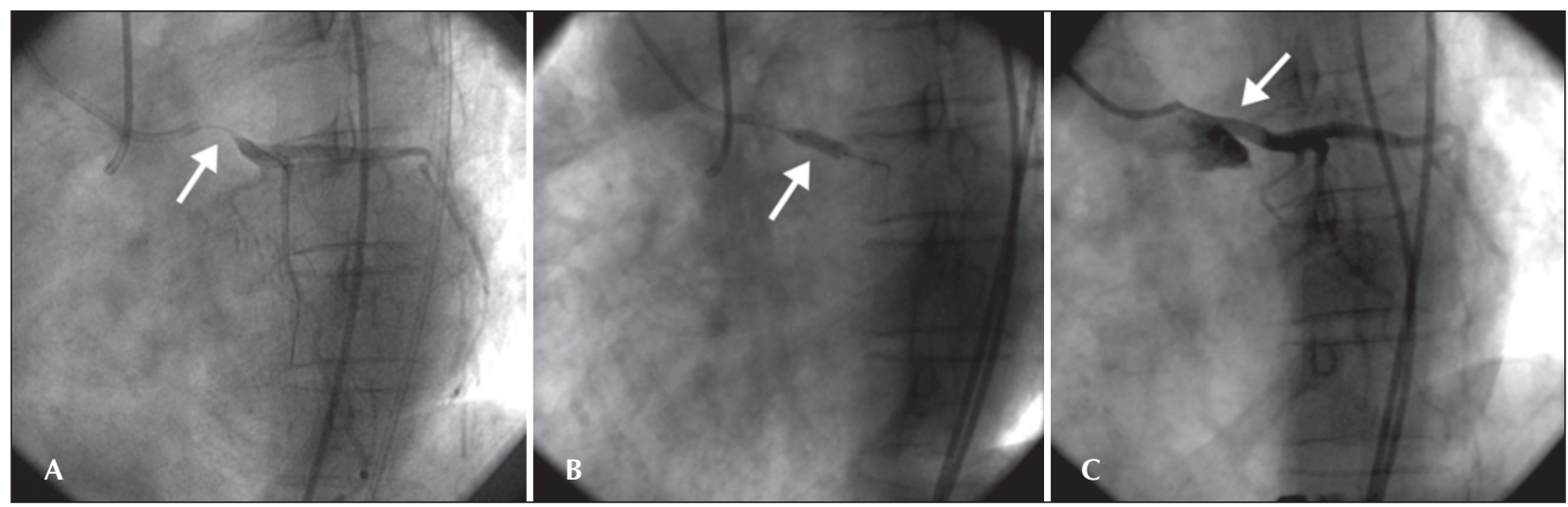

Figura 3 - Em A, tronco de coronária esquerda em oblíqua anterior esquerda com fio guia passado, antes da dilatação (seta). Em B, tronco de coronária esquerda em oblíqua anterior esquerda, com balão insuflado (seta). Em C, tronco de coronária esquerda em oblíqua anterior esquerda, após o implante do stent (seta).
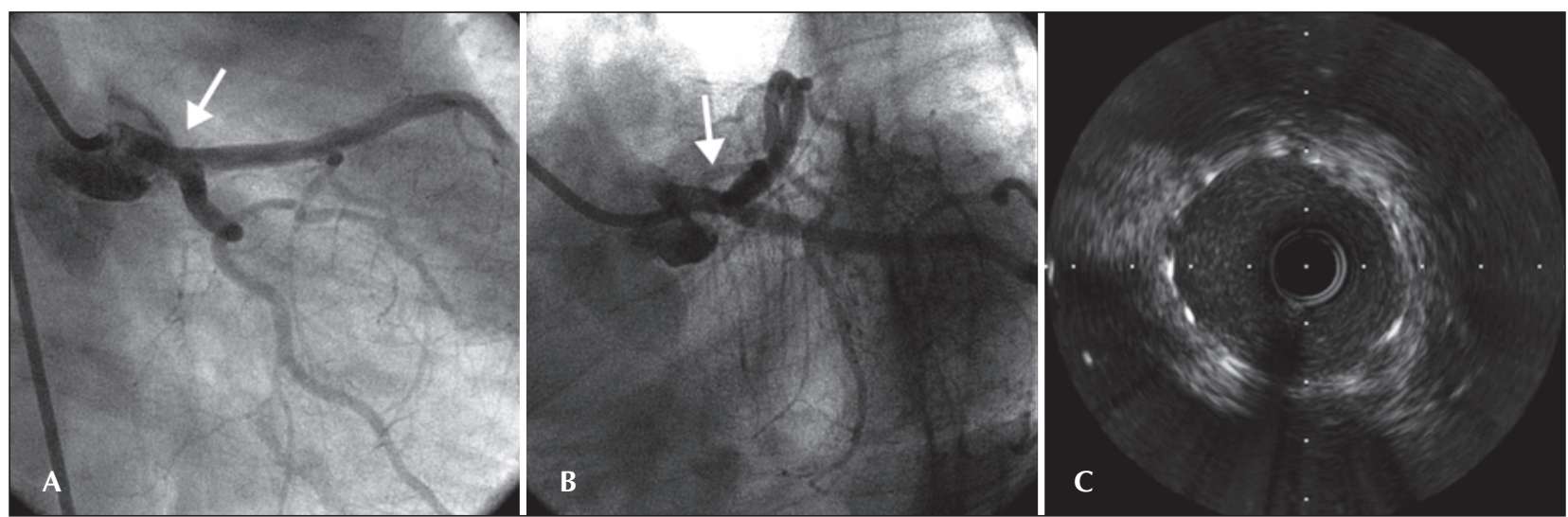

Figura 4 - Em A, tronco de coronária esquerda livre de obstrução, em oblíqua anterior direita (seta). Em B, tronco de coronária esquerda livre de obstrução, em oblíqua anterior esquerda caudal (seta). Em C, ultrassom intracoronário com proliferação neointimal mínima e sem sinais de aterosclerose subjacente.

As opções terapêuticas para a compressão extrínseca do tronco de coronária esquerda por dilatação do tronco de artéria pulmonar incluem tratamento clínico, intervenção percutânea, correção cirúrgica da cardiopatia congênita, cirurgia de revascularização miocárdica ou transplante cardíaco. Aproximadamente 16\% dos casos publicados foram tratados com cirurgia de revascularização miocárdica ou com combinação de cirurgia de revascularização miocárdica e correção da cardiopatia congênita. Dos seis casos de hipertensão arterial pulmonar idiopática, ,10,13-16 três foram submetidos a intervenção percutânea com stent convencional ou farmacológico, com bom resultado clínico e angiográfico, no seguimento por seis meses; dois seguiram em tratamento clínico; e um paciente foi submetido a transplante coração-pulmão. Em todos os casos os pacientes apresentavam angina do peito e o diagnóstico só foi possível pelo estudo hemodinâmico/ angiográfico, a que foram submetidos para quantificar e definir a etiologia da hipertensão arterial pulmonar. Em nenhum caso foi indicado o cateterismo para investigação de isquemia coronária.
A história natural da doença ainda não foi esclarecida e a melhor estratégia terapêutica ainda é tema de discussão. A hipertensão arterial pulmonar representa um preditor de mortalidade em pacientes submetidos a cirurgia de revascularização miocárdica, pela falência do ventrículo direito no pós-operatório. ${ }^{17,18}$ Por outro lado, o implante de stent em tronco de coronária esquerda não protegido, nos pacientes com doença aterosclerótica, também cursa com elevada morbidade, ${ }^{19}$ mesmo quando realizado em centros especializados, em pacientes de alto risco.

Em 2001, Rich et al. ${ }^{20}$ relataram dois casos de compressão extrínseca de tronco de coronária esquerda por dilatação do tronco de artéria pulmonar em pacientes com hipertensão arterial pulmonar primária, tratados com epoprostenol endovenoso por 30 dias, para estabilização clínica, e posteriomente submetidos a implante de stent intracoronário. Os autores, ao identificar resultados angiográficos satisfatórios, meIhora dos sintomas e normalização da fração de ejeção ( $<25 \%$ antes do procedimento), porém sem redução 
Mendes AA, et al. Tratamento com Stent em Tronco de Artéria Coronária Esquerda por Compressão do Tronco da Artéria Pulmonar em Paciente com Hipertensão Pulmonar Esquistossomótica. Rev Bras Cardiol Invasiva. 2010;18(1):89-94.

das pressões e do diâmetro arterial pulmonar, sugeriram que seria pouco provável o mesmo resultado do tratamento intervencionista caso o paciente tivesse recebido tratamento clínico isolado.

Atualmente, o tratamento das lesões de tronco de artéria coronária esquerda por via percutânea, por meio dos stents, tem se mostrado viável e motivo de estudos, principalmente após se ter estabelecido a segurança dos stents com eluição de antiproliferativos. ${ }^{21}$

Um capítulo à parte diz respeito às lesões de tronco de artéria coronária esquerda de etiologia nãoaterosclerótica, mas por compressão extrínseca, como no caso apresentado, diagnosticado e tratado em centro especializado no tratamento de hipertensão pulmonar, onde, não tão raramente, se observa algum grau de compressão do tronco de coronária esquerda por artéria pulmonar dilatada. Não há consenso acerca do stent mais adequado nesse cenário, questionando-se entre o convencional e o farmacológico, uma vez que o processo patogênico não diz respeito à disfunção endotelial ou aos processos inflamatórios locais. Temos um endotélio em seu perfeito estado funcional, o que poderia ser traduzido em bons resultados com o uso do stent convencional. É importante citar que a técnica do implante compreende completa aposição das hastes e ganho luminal máximo, guiado pela ultrassonografia intracoronária, como no caso apresentado.

Sendo este o primeiro caso com sucesso de stent convencional para tratamento de tronco de coronária esquerda decorrente de compressão pelo tronco de artéria pulmonar em pacientes com hipertensão arterial pulmonar de etiologia esquistossomótica, a revascularização percutânea mostrou ser uma opção nesse grupo de pacientes, com alívio dos sintomas e melhora da classe funcional. No entanto, não dispomos ainda de dados sobre o benefício a longo prazo, sendo necessário estudo de maior número de pacientes.

\section{CONCLUSÃO}

Em pacientes com hipertensão arterial pulmonar e angina do peito, a compressão coronária deverá ser considerada, sendo necessário que esses pacientes sejam submetidos a avaliação da anatomia coronária. A isquemia ventricular esquerda decorrente de compressão do tronco coronário pode ser o mecanismo de dor precordial, disfunção ventricular esquerda e morte súbita em pacientes com hipertensão arterial pulmonar, para os quais não se tenha ainda firmado diagnóstico. O tratamento percutâneo com implante de stent, guiado por ultrassom intracoronário, pode ser realizado em centros especializados, com bons resultados angiográficos e clínicos, porém na literatura ainda não há consenso a respeito de qual stent, convencional ou farmacológico, utilizar nesse cenário.

\section{CONFLITO DE INTERESSES}

Os autores declararam inexistência de conflito de interesses relacionado a este manuscrito.

\section{REFERÊNCIAS}

1. Rubin LJ. Primary pulmonary hypertension. N Engl J Med. 1997;336:111-7.

2. Rich S, Dantzker DR, Ayres S, Bergofsky EH, Brundage BH, Detre KM, et al. Primary pulmonary hypertension: a national prospective study. Ann Intern Med. 1987;107:216-33.

3. Viar W, Harrison T. Chest pain in association with pulmonary hypertension: its similarity to the pain due to coronary disease. Circulation. 1952;5:1-11.

4. Vlhakes G, Turley K, Hoffman J. The pathophysiology of failure in acute right ventricular hypertension: hemodynamic and biochemical correlation. Circulation. 1981;63:87-95.

5. Andrade AZ, Andrade SG. Pathogenesis of schistosomiasis pulmonary arteritis. Am J Trop Med Hyg. 1970;19:305-10.

6. Jawahiry KI, Karpas CM. Pulmonary schistosomiasis: a detailed clinicopathologic study. Am Rev Resp Dis. 1963;88:517-27.

7. Watt G, Long GW, Calubaquib C. Cardiopulmonary involvement rare in severe Schistosoma japonicum infection. Trop Geogr Med. 1986;38:233-9.

8. Rocha RL, Pedroso ERP. Forma pulmonar crônica da esquistossomose mansônica. Avaliação clínico-cardiológica. Rev Soc Bras Med Trop. 1990;23:83-9.

9. Bijl M, Bronzwaer JG, van Rossum AC, Verheugt FW. Angina pectoris due to left main coronary compression in Eisenmenger ductus arteriosus. Am Heart J. 1993;125:1767-71.

10. Patrat JF, Jondeau G, Dubourg $O$, Lacombe $P$, Rigaud $M$, Bourdarias J, et al. Left main coronary artery compression during primary pulmonary hypertension. Chest. 1997;112: 842-3.

11. Corday E, Gold H, Kaplan L. Coronary artery compression: an explanation for the cause of coronary insufficiency in pulmonary hypertension. Trans Am Coll Cardiol. 1957;7: 93-103.

12. Piña Y, Exaire JE, Sandoval J. Left main coronary artery extrinsic compression syndrome: a combined intravascular ultrasound and pressure wire report. J Invasive Cardiol. 2006; 18:1042-45.

13. Kajita LJ, Martinez EE, Ambrose JA, Lemos PA, Esteves A, Nogueira da Gama M, et al. Extrinsic compression of the left main coronary artery by a dilated pulmonary artery: clinical, angiographic, and hemodynamic determinants. Catheter Cardiovasc Interv. 2001;52:49-54.

14. Mesquita SM, Castro CR, Ikari NM, Oliveira SA, Lopes AA. Likelihood of left main coronary artery compression based on pulmonary trunk diameter in patients with pulmonary hypertension. Am J Med. 2004;116:369-74.

15. Kawut SM, Silvestry FE, Ferrari VA, DeNofrio D, Axel L, Loh $\mathrm{E}$, et al. Extrinsic compression of the left main coronary artery by the pulmonary artery in patients with long-standing pulmonary hypertension. Am J Cardiol. 1999;83:984-6.

16. Varela SG, Montes Orbe PM, Villa JA, Egurbide MV, Sainz I, Benguría JIB. Stenting in primary pulmonary hypertension with compression of the left main coronary artery. Rev Esp Cardiol. 2004;57:695-8.

17. Reich DL, Bodian CA, Krol M, Kuroda M, Osonki T, Thys DM. Intraoperative hemodynamic predictors of mortality, stroke and myocardial infarction after coronary artery bypass surgery. Anesth Analg. 1999;89:814-22. 
Mendes AA, et al. Tratamento com Stent em Tronco de Artéria Coronária Esquerda por Compressão do Tronco da Artéria Pulmonar em Paciente com Hipertensão Pulmonar Esquistossomótica. Rev Bras Cardiol Invasiva. 2010;18(1):89-94.

18. Kuralay E, Demirkilic U, Oz BS, Cíngöz F, Tatar H. Primary pulmonary hypertension and coronary artery bypass surgery. J Card Surg. 2002;17:79-80.

19. Park SJ, Park SW, Hog MK, Lee CW, Lee JJ, Kim JJ. Longterm (three-year) outcomes after stenting of unprotected left main coronary artery stenosis in patients with normal left ventricular function. J Am Coll Cardiol. 2003;91:12-6.
20. Rich S, McLaughlin VV, O'Neill W. Stenting to reverse left ventricular ischemia due to left main coronary compression in primary pulmonary hypertension. Chest. 2001;120:1412-5.

21. Serruys PW, Morice MC, Kappetein AP, Colombo A, Holmes DR, Mack MJ, et al. Percutaneous coronary intervention versus coronary-artery bypass grafting for severe coronary artery disease. N Engl J Med. 2009;360:961-72. 